\title{
Factores climático determinantes del rendimiento y la producción de papa en el distrito de Juli, Puno-Perú, 2000-2018
}

Climatic factors determining potato yield and production in the Juli district, Puno - Peru, 2000-2018

Fatores climáticos que determinam o rendimento e a produção da batata no distrito de Juli, Puno - Peru, 2000-2018

\author{
Julio Cesar Quispe Mamani \\ jcquispe@unap.edu.pe \\ https://orcid.org/0000-0002-3938-1459 \\ Universidad Nacional del Altiplano, Puno-Perú
}

Edson Apaza Mamani

eapaza@unap.edu.pe

https://orcid.org/0000-0001-9169-077X

Universidad Nacional del Altiplano, Puno-Perú

Omar Henry Marca Flores

biomarca78@hotmail.com

https://orcid.org/0000-0003-2339-501X

Universidad Nacional del Altiplano, Puno-Perú

\author{
Dominga Asunción Calcina Álvarez \\ dcalcina@unamad.edu.pe \\ https://orcid.org/0000-0002-6869-0939
}

Universidad Nacional Amazónica de Madre de Dios, Puerto Maldonado-Perú

\author{
Eliseo Pumacallahui Salcedo \\ eliseo.pumacallahui@uniq.edu.pe \\ https://orcid.org/0000-0002-5730-684X \\ Universidad Nacional Intercultural de Quilabamba, \\ Cusco-Perú
}

\author{
Cristóbal Rufino Yapuchura Saico \\ cyapuchura@unap.edu.pe \\ https://orcid.org/0000-0003-1956-3922
}

Universidad Nacional del Altiplano, Puno-Perú

Artículo recibido 28 de octubre 2021 / Arbitrado y aceptado 26 de noviembre 2021 / Publicado 13 de diciembre 2021

\section{RESUMEN}

El objetivo fue encontrar los factores climáticos que determinan el rendimiento y la producción de papa en el distrito de Julia, entre los periodos 2000-2018, para lo cual se utilizó la información estadística de la Dirección Regional Agraria Puno y del Servicio Nacional de Meteorología e Hidrología de Puno. Se utilizó la metodología descriptiva y correlacional, considerando el modelo econométrico de tipo log-log. Se determinó que las variables climáticas como la temperatura mínima, la temperatura máxima determinan de manera positiva en el rendimiento de la producción y en la producción de papa y las precipitaciones de la lluvia determinan de manera negativa en el rendimiento de la producción y en la producción de papa. Finalmente, la temperatura mínima, la temperatura máxima y las precipitaciones explican al rendimiento de la producción y en la producción de papa en $97.96 \%$ y 97.95\% respectivamente.

Palabras clave: Cambio climático; Producción agrícola; Rendimiento; Zona rural

\section{ABSTRACT}

The objective was to find the climatic factors that determine the yield and potato production in the Julia district, between the periods 2000-2018, for which statistical information from the Puno Regional Agrarian Directorate and the National Meteorology Service were used. Hydrology of Puno. The descriptive and correlational methodology was used, considering the log-log type econometric model. It was determined that climatic variables such as the minimum temperature, the maximum temperature positively determine the production yield and potato production, and the rainfall precipitations negatively determine the production yield and production. of potato. Finally, the minimum temperature, the maximum temperature and the rainfall explain the production yield and potato production in $97.96 \%$ and $97.95 \%$ respectively.

Key words: Climate change; Agricultural production; Yield; Rural area

\section{RESUMO}

O objectivo era encontrar os factores climáticos que determinam o rendimento e a produção de batata no distrito de Julia, entre os períodos 2000-2018, para os quais se utilizou informação estatística da Direcção Agrária Regional de Puno e do Serviço Nacional de Meteorologia Hidrologia de Puno. Foi utilizada a metodologia descritiva e correlacional, considerando o modelo econométrico do tipo log-log. Determinou-se que as variáveis climáticas como a temperatura mínima, a temperatura máxima determina positivamente o rendimento da produção e a produção da batata, e as precipitações pluviométricas determinam negativamente o rendimento da produção e a produção. Finalmente, a temperatura mínima, a temperatura máxima e a precipitação explicam o rendimento da produção e a produção de batata em $97,96 \%$ e $97,95 \%$ respectivamente.

Palavras-chave: Mudança climática; Produção agrícola; Desempenho; Área rural 


\section{INTRODUCCIÓN}

En los últimos años los cambios climáticos han estado afectando a los sistemas físicos y biológicos, esto se da en el sector de la agricultura, se dice que esto se da en las tendencias generales del incremento de la temperatura dado las perturbaciones en el régimen pluviométrico (1-6).

En Latinoamérica y el Caribe la agricultura es una de las actividades económicas más importantes, ya que genera un porcentaje destacado del total del Producto Bruto Interno (PBI) en la región. De tal manera que, se ha observado que la agricultura, el uso del suelo y los cambios de uso de este representaron dos tercios de las emisiones totales de gases de efecto invernadero en la región, es decir el cambio climático está directamente relacionado (7-14).

Sin embargo, los espacios internacional y global, la Organización de las Naciones Unidas (ONU) para la Ingesta de alimentos y la agricultura ha desarrollado el término de agricultura climáticamente inteligente, el cual se apoya en un enfoque para desarrollar las condiciones técnicas, de políticas e inversión, asimismo, la mitigación del calentamiento global puede ser afectada por políticas y tendencias socioeconómicas de carácter general, como las que se relacionan con desarrollo, la sostenibilidad y la igualdad (8,15-21).

Estudios, llevados a cabo en el 2001 y bajo la órbita del Panel Intergubernamental del cambio climático (IPCC), estimaron que la agricultura enfrentará nuevos desafíos en las próximas décadas (2,22-24). Desde un punto de vista amplio, la conjunción de amenazas y vulnerabilidad produce peligros climáticos relevantes no solamente para los agricultores, las sociedades rurales y la zona agrícola sino además para el logro de fines de desarrollo en general, incluida el decrecimiento de la pobreza, la entrada a la enseñanza, la nutrición y el agua, el riego y la electricidad, mejoras de infraestructuras y aumento de los ingresos y las exportaciones por la agricultura (6,25-27).

El Presidente de Panel Intergubernamental de Cambio Climático (IPCC) quien fue premio Nobel de la paz 2007, nos da a entender que el Perú es uno de los países más vulnerables a los efectos del cambio climático por lo cambios en las cosechas, entre otros, por lo tanto está afectando a la economía del país $(12,28)$.

Por su parte, el director de la Dirección General de Asuntos Ambientales (DGAA) del MINAGRI, dio a conocer que el Perú está dentro de los diez países más vulnerables dentro de todo el mundo, esto se debe al cambio climático. Añadiendo un ejemplo; el $71 \%$ de la población peruana está situada en áreas expuestas al alto peligro. Como también más de 15 mil hectáreas se pierden en cada campaña agrícola que se realiza, estos vienen asociada a eventos climáticos, y en las últimas doce campañas agrícolas que se dio el Estado peruano ha podido contabilizar pérdidas por razones de clima por un valor de S/. 2,597 millones (29).

Si bien es cierto actualmente en el Perú, la Papa es el principal cultivo en la superficie sembrada, la cual representa el $25 \%$ del PBI 
agropecuario. Es la base de la alimentación de la zona andina y es producido por $600 \mathrm{mil}$ pequeñas unidades agrarias a nivel de todo el Perú $(12,30,31)$.

En el altiplano peruano, los eventos climáticos adversos son muy frecuentes como las heladas y sequias, las cuales son quienes afectan a las actividades productivas como agricultura y ganadería, presenta un peligro ya que estas son las que velan las principales fuentes de ingreso a muchas familias (32).

El principal cultivo de los pequeños agricultores de la región andina es la papa, esto es porque existe gran diversidad genética de especies de papas cultivadas y especies de papas silvestres, así como también la fuente importante de calorías y proteínas (13). A pesar del alto potencial que existe del rendimiento del cultivo de la papa, en el Perú, especialmente que se sitúa en el Altiplano de Puno, el promedio de la producción y productividad es baja, en comparación a la existencia del rendimiento promedio de otros departamentos. En la actualidad en Puno hay un aproximado de 40000 hectáreas sembradas de papa, el cual constituye un volumen de producción de 225000 toneladas y un rendimiento promedio de $9,6 \mathrm{t} / \mathrm{ha}$ (33).

En las condiciones agroecológicas del distrito de Juli como parte del Altiplano de Puno, el factor más importante que limita los niveles de producción y productividad de la papa es el clima adverso por la presencia de heladas, sequías, granizadas, irregular distribución de precipitaciones pluviales, el 90\% del área aproximada sembrada con papa en Puno, está expuesta a heladas y el $98 \%$ se siembra bajo secano o bajo condiciones de lluvias (34). Por estas razones, se requieren variedades de papa; que además de tener buen potencial de rendimiento y comportamiento en las condiciones agroecológicas del altiplano de Puno, presenten resistencia o tolerancia a factores abióticos y bióticos, y buena calidad $(13,33)$.

Por lo cual, la investigación busca responder las siguientes interrogantes: ¿Cuáles son los factores climáticos que determinan el rendimiento y la producción de papa en el distrito de Julia, entre los periodos 2000-2018? En este sentido el objetivo de la investigación fue encontrar los factores climáticos que determinan el rendimiento y la producción de papa en el distrito de Julia, entre los periodos 2000-2018.

\section{MATERIALES Y MÉTODOS}

El estudio se realizó en la zona productora de papa, del distrito de Juli, provincia de Chucuito, departamento de Puno; el cual se encuentra ubicado sobre los $3869 \mathrm{msnm}$ (Figura 1). Para el estudio de este trabajo se tomaron datos estadísticos de la Dirección Regional Agraria de Puno (DRA), campaña agrícola 2000-2018 del rendimiento y la producción de papa. La información de las variables agroclimáticas como temperaturas máximas y mínimas expresadas en grados Celsius $\left({ }^{\circ} \mathrm{C}\right)$, precipitaciones en milímetros $(\mathrm{mm} / \mathrm{mc})$, han sido proporcionados por el Servicio Nacional de Meteorología e Hidrología (35). 


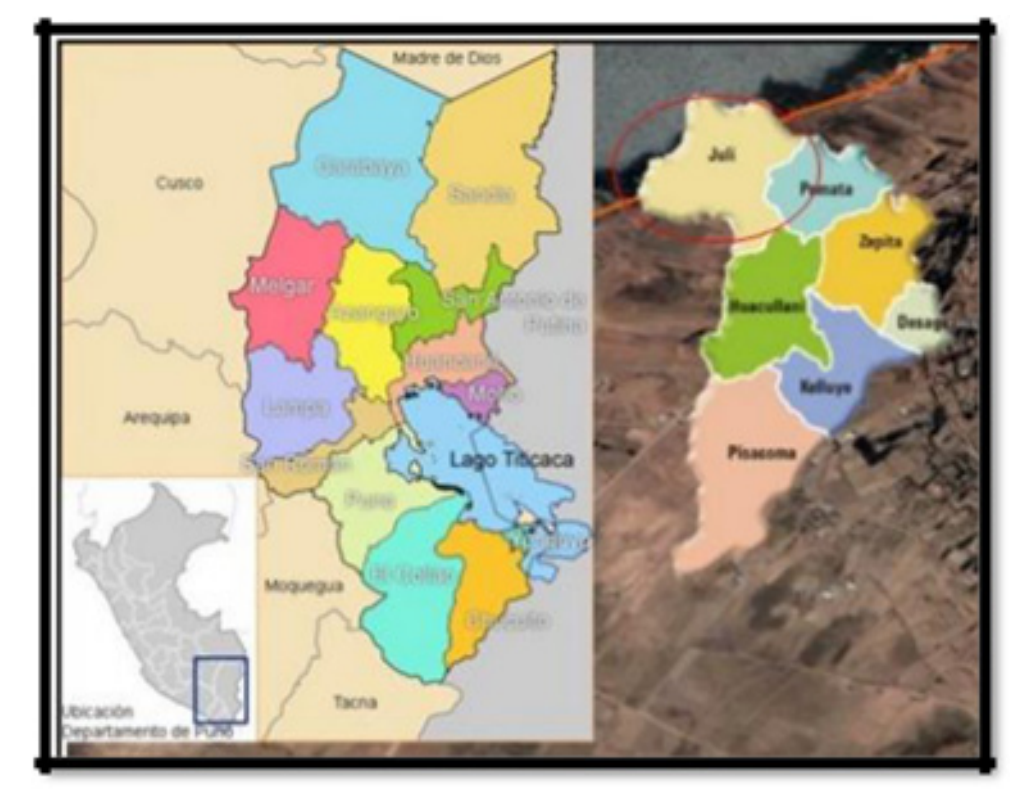

Figura 1. Ubicación geográfica del área de estudio.

Este estudio correspondió a un enfoque cuantitativo (36), que tuvo como objetivo determinar el efecto del cambio climático en el rendimiento y producción de papa, en el distrito de Juli (2000 - 2018) mediante el uso de la relación econométrica lineal y cuadrática, para lo cual se utilizó las siguientes variables que se detallan en la Tabla 1.

Tabla 1. Operacionalización de variables del modelo.

\begin{tabular}{lccc}
\hline \multicolumn{1}{c}{ Variable } & Indicador & Unidad de medida & Fuente de información \\
\hline Variable dependiente & & & \\
Rendimiento de producción & Índice & $\mathrm{Kg} . / \mathrm{Ha}$ & $\mathrm{DRA}$ \\
Producción de papa & Toneladas & Toneladas/Ha & DRA \\
Variables Independientes & & & \\
Precipitación de lluvia & $\mathrm{mm}$ & $\mathrm{mm}$ & $\mathrm{SENAMHI}$ \\
Temperatura máxima del ambiente & Celsius & ${ }^{\circ} \mathrm{C}$ & $\mathrm{SENAMHI}$ \\
Temperatura mínima del ambiente & Celsius & ${ }^{\circ} \mathrm{C}$ & $\mathrm{SENAMHI}$ \\
\hline
\end{tabular}

\section{Modelo econométrico}

El modelo econométrico propuesto para analizar los factores que influyen en el rendimiento y la producción de papa fue de tipo Log-Log como se muestra a continuación:

LogRendimiento de la producción $\mathrm{n}_{\mathrm{t}}=\mathrm{a}_{0}+\mathrm{a}_{1}$ *

LogTemperatura máxima del ambiente ${ }_{\mathrm{t}}+\mathrm{a}_{2} *$

LogTemperatura mínima del ambiente $-a_{3} *$ LogPrecipitación de lluvia ${ }_{t}$

LogProducción de papa $\mathrm{a}_{\mathrm{t}}=\mathrm{a}_{0}+\mathrm{a}_{1} *$ LogTemperatura máxima del ambiente $\mathrm{t}_{\mathrm{t}}+\mathrm{a}_{2}$ *

LogTemperatura mínima del ambiente $-a_{3} *$ LogPrecipitación de lluvia $a_{t}$ 


\section{RESULTADOS Y DISCUSIÓN}

Comportamiento de los factores climáticos, rendimiento y la producción de papa durante el período 2000-2018

El comportamiento de las precipitaciones, la temperatura máxima y mínima del ambiente, el rendimiento y la producción de la papa se detalla en la siguiente figura, donde la producción de papa en el distrito de Juli, desde el año 2000 hasta 2018 fue muy consistente, en vista que dicha producción desde el año 2006
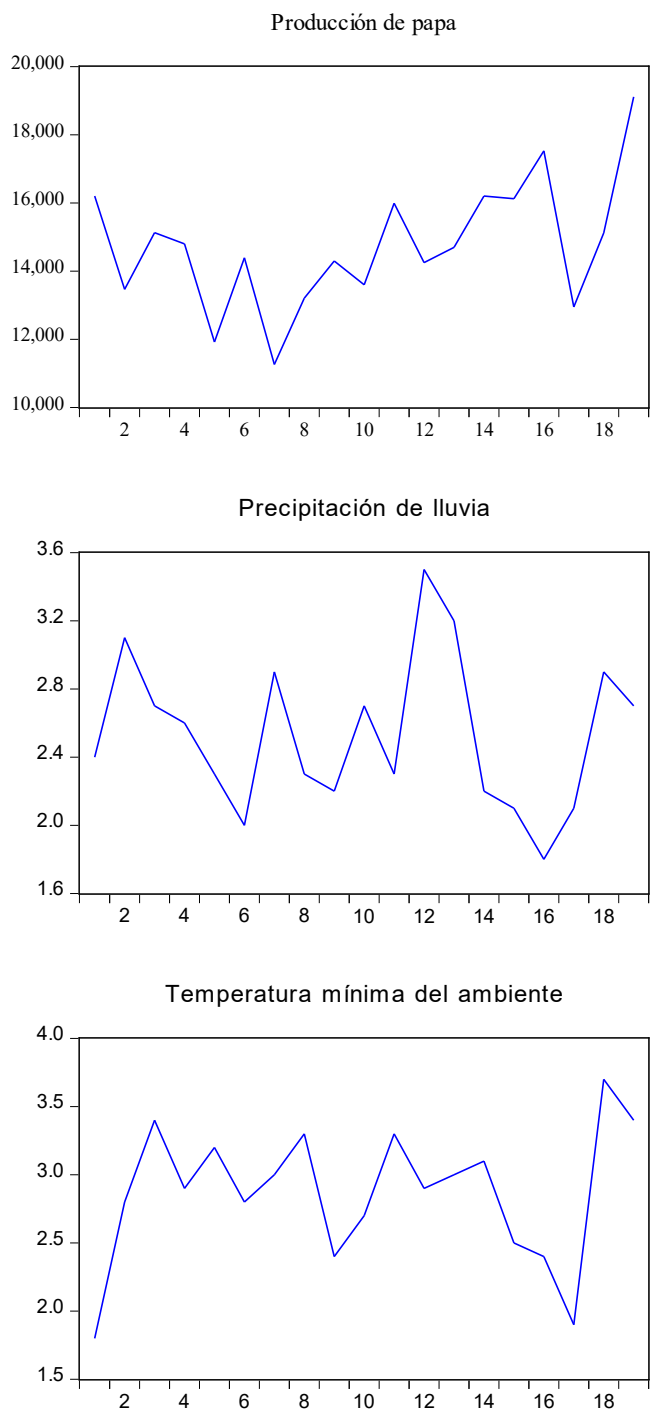

se mantuvo creciente hasta llegar al pico más alto el año 2015 con una producción de 17,522 toneladas de papa, para luego en el año 2016 decreció de manera significativa, de ese modo para los años 2017 y 2018 la producción de papa aumento a gran escala (Figura 2); alcanzando un promedio de producción de 14,746.26 toneladas por año, llegando a producir un volumen máximo de 19,111.00 toneladas y un valor mínimo de $11,260.00$ toneladas por campaña agrícola y una desviación estándar de 1,886.56 toneladas por año (Tabla 2).
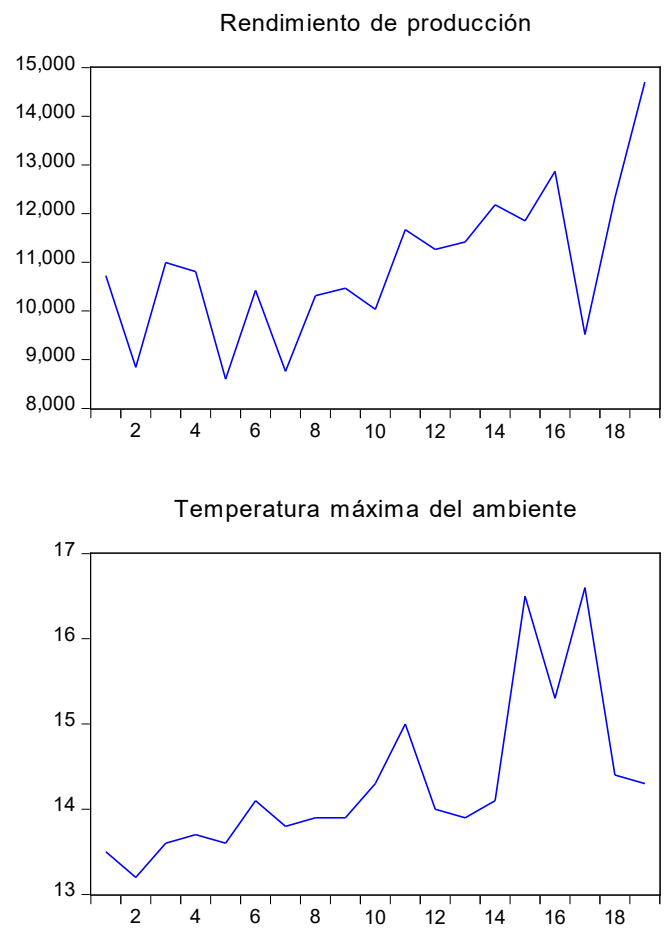

Figura 2. Comportamiento de las variables en análisis. 
En el caso del rendimiento de la producción de papa desde el año 2006 hasta el 2015 se mantuvo en crecimiento, llegando a un rendimiento $12,864.904 \mathrm{Kg}$. / Ha, para a inicios del año 2016 el rendimiento fue decreciente, de tal modo que desde inicios del año 2017 tuvo una respuesta creciente (Figura 2). Es por lo cual que, el rendimiento promedio por campaña agrícola fue de 10,935.29 $\mathrm{Kg}$. / $\mathrm{Ha}$, alcanzando un rendimiento máximo de $14,700.77 \mathrm{Kg}$. / Ha y un rendimiento mínimo de 10, 808.62 Kg. / Ha, con una desviación estándar de 1,516.22 Kg. / Ha (Tabla 2).

En el caso de la temperatura que más altas llego el distrito de Juli fue en los años 2014 con $16.50^{\circ} \mathrm{C}$ y $201616.60^{\circ} \mathrm{C}$ (Figura 2). Además, complementariamente en promedio la temperatura en dicha zona en el periodo de análisis fue de $14.30{ }^{\circ} \mathrm{C}$ (Tabla 2). Respecto al comportamiento de la temperatura mínima en el distrito de Juli, la más bajas ha llegado fue a inicios del año 2000 con $1.80^{\circ} \mathrm{C}$, asimismo se registró en los años 2014 con $2.40^{\circ} \mathrm{C}$ y 2016 con $1.80^{\circ} \mathrm{C}$ respectivamente (Tabla 2 ).

Sobre el comportamiento de las precipitaciones en el distrito de Juli en el periodo de análisis, esta tuvo un comportamiento notorio y muy creciente en el primer trimestre de todos los años, resaltando en su mayor proporción en el año 2011 (Figura 2); la misma que tuvo en promedio el valor de $2.53 \mathrm{~mm}$, con un valor máximo de $3.50 \mathrm{~mm}$ y un valor mínimo de 1.80 $\mathrm{mm}$ (Tabla 2).

Tabla 2. Operacionalización de variables del modelo.

\begin{tabular}{lccccc}
\hline \multicolumn{1}{c}{ Estadístico } & $\begin{array}{c}\text { Producción } \\
\text { de papa }\end{array}$ & $\begin{array}{c}\text { Rendimiento } \\
\text { de producción }\end{array}$ & $\begin{array}{c}\text { Precipitación } \\
\text { de lluvia }\end{array}$ & $\begin{array}{c}\text { Temperatura } \\
\text { máxima del } \\
\text { ambiente }\end{array}$ & $\begin{array}{c}\text { Temperatura } \\
\text { mínima del } \\
\text { ambiente }\end{array}$ \\
\hline Media & $14,746.26$ & $10,935.29$ & 2.53 & 14.30 & 2.87 \\
Mediana & $14,696.00$ & $10,808.62$ & 2.40 & 14.00 & 2.90 \\
Valor máximo & $19,111.00$ & $14,700.77$ & 3.50 & 16.60 & 3.70 \\
Valor mínimo & $11,260.00$ & $8,602.89$ & 1.80 & 13.20 & 1.80 \\
Desviación estándar & $1,886.56$ & $1,516.22$ & 0.45 & 0.93 & 0.50 \\
Kurtosis & 3.10 & 3.31 & 2.43 & 4.25 & 2.81 \\
Jarque-Bera & 0.32 & 0.85 & 0.95 & 8.10 & 1.13 \\
Probabilidad & 0.85 & 0.65 & 0.62 & 0.02 & 0.57 \\
\hline
\end{tabular}

Relación entre la producción, rendimiento con las variables que las determinan

La relación existente entre las precipitaciones de la lluvia con la producción y el rendimiento de la papa es inversa; es decir que, ante un incremento de las precipitaciones de la lluvia, entonces la producción y el rendimiento tienden a disminuir, el cual es corroborado por el diagrama de dispersión de estas variables (Figura 3), donde se muestra que existe una relación negativa o indirecta entre dichas variables, además que muestran 
un $\rho$ de Pearson igual a -0.211 y -0.081 , correspondiente a una correlación negativa baja (Tabla 3).

En el caso de la relación existente entre la temperatura máxima con la producción y el rendimiento de la papa es directa; es decir que, ante un incremento de la temperatura máxima, entonces la producción y el rendimiento tienden a aumentar, el cual es corroborado por el diagrama de dispersión de estas variables (Figura 3), donde se muestra que existe una relación positiva o directa entre dichas variables, además que muestran un $\rho$ de Pearson igual a 0.220 y 0.257 , correspondiente a una correlación positiva baja (Tabla 3).
Consecuentemente, la relación existente entre la temperatura mínima con la producción y el rendimiento de la papa al igual que en el caso anterior es directa; es decir que, ante un incremento de la temperatura mínima, entonces la producción y el rendimiento tienden a incrementar, el cual es corroborado por el diagrama de dispersión de estas variables (Figura 3), donde se muestra que existe una relación positiva o directa entre dichas variables, además que se evidencia un $\rho$ de Pearson igual a 0.031 y 0.251 , correspondiente a una correlación positiva baja (Tabla 3).

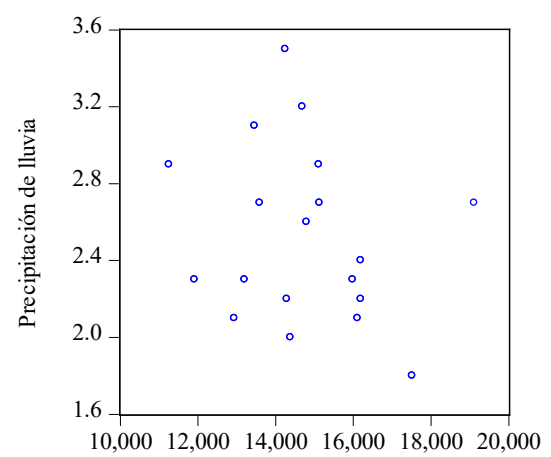

Producción de papa

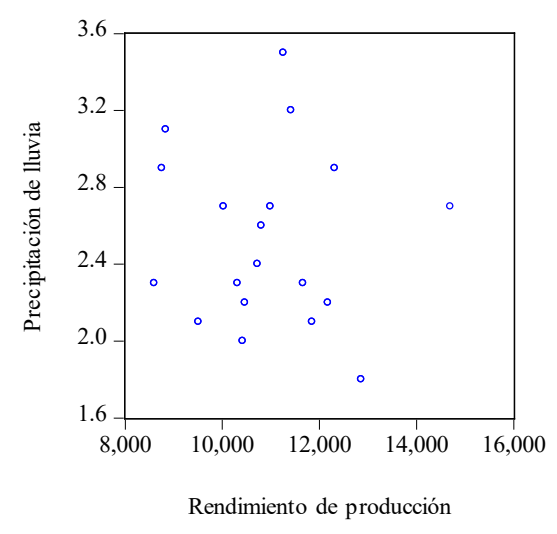

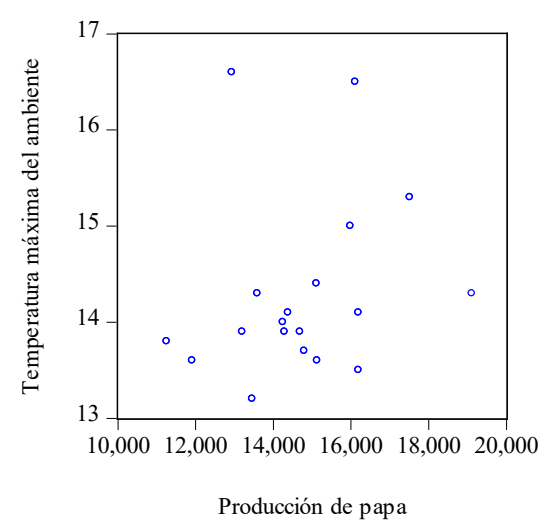

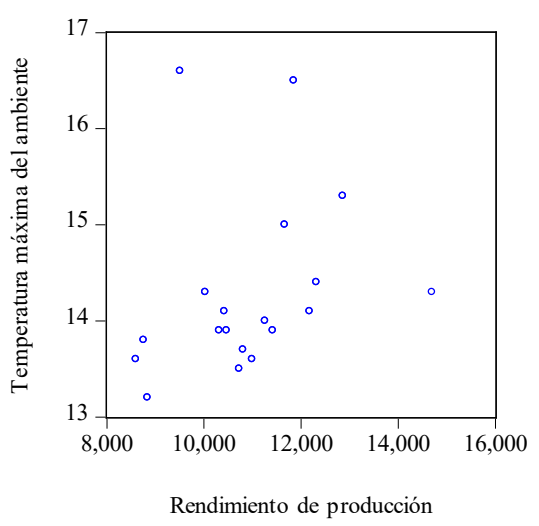

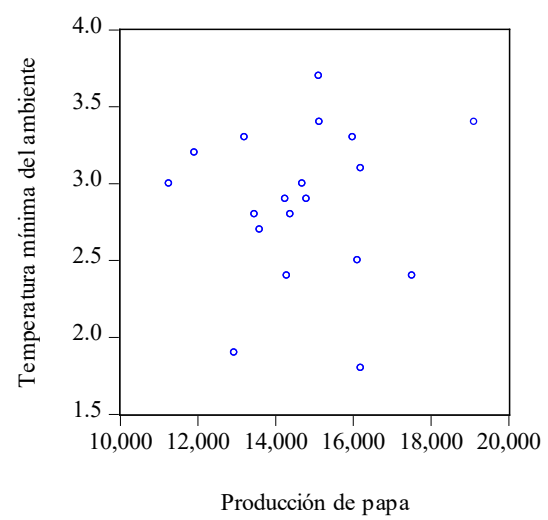

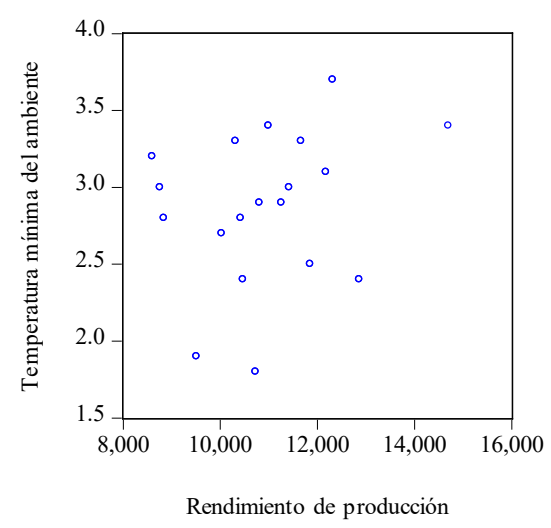

Figura 3. Relación entre el comportamiento de la producción y rendimiento con sus determinantes. 
Tabla 3. Matriz de correlación entre la producción, el rendimiento y sus determinantes.

\begin{tabular}{lccccc}
\hline \multicolumn{1}{c}{ Estadístico } & $\begin{array}{c}\text { Producción } \\
\text { de papa }\end{array}$ & $\begin{array}{c}\text { Rendimiento } \\
\text { de producción }\end{array}$ & $\begin{array}{c}\text { Precipitación } \\
\text { de lluvia }\end{array}$ & $\begin{array}{c}\text { Temperatura } \\
\text { máxima del } \\
\text { ambiente }\end{array}$ & $\begin{array}{c}\text { Temperatura } \\
\text { mínima del } \\
\text { ambiente }\end{array}$ \\
\hline Producción de papa & 1.000 & 0.925 & -0.211 & 0.220 & 0.031 \\
Rendimiento de producción & 0.925 & 1.000 & -0.081 & 0.257 & 0.251 \\
Precipitación de lluvia & -0.211 & -0.081 & 1.000 & -0.483 & 0.365 \\
Temperatura máxima del ambiente & 0.220 & 0.257 & -0.483 & 1.000 & -0.351 \\
Temperatura mínima del ambiente & 0.031 & 0.251 & 0.365 & -0.351 & 1.000 \\
\hline
\end{tabular}

Análisis de factores climáticos que más influyen en el rendimiento y la producción de papa en el Distrito de Juli (2000-2018)

Para el contraste de la hipótesis se planteó la hipótesis de investigación que es la siguiente: Ha: Los factores determinantes de la producción de papa y el rendimiento de la producción son precipitación de lluvia temperatura máxima del ambiente, temperatura mínima del ambiente; por lo que para el contraste de la misma se realizó las estimaciones econométricas mediante el modelo Log-Log, donde los resultados obtenidos se muestran en la Tabla 4.

Analizando las estimaciones para el rendimiento de la producción de papa, en la Tabla 4 se puede ver que la variable que más influye o mejor explica es la temperatura mínima, seguido por la temperatura máxima y las precipitaciones. Se obtuvo un R cuadrado ajustado de $97.96 \%$, demostrando que las variables ambientales explican en $97.96 \%$ al rendimiento de la producción, siendo un modelo muy eficiente.

Tabla 4. Estimación del modelo para el Rendimiento de la papa en el Distrito de Juli.

\begin{tabular}{lcc}
\hline \multicolumn{1}{c}{ Variables } & Rendimiento de la producción & Producción de papa \\
\hline Temperatura máxima & 0.319106 & 0.30565 \\
Temperatura mínima & 0.4431514 & 0.3180097 \\
Precipitaciones & -0.3192216 & -0.399532 \\
$\mathbf{R}^{2}$ & 0.98997 & 0.9896 \\
$\mathbf{R}^{2}$ ajustado & 0.9796 & 0.9795 \\
F stat. & 15658.44 & 12922.97 \\
Prob F & 0.0000 & 0.0000 \\
\hline
\end{tabular}


En el caso del nivel de significancia individual, estas son menores a $5 \%$, siendo consistente cada uno de las variables, determinando que ante un incremento de la temperatura mínima es la zona de estudio en $1 \%$, entonces el rendimiento de la producción de papa incrementara en $44.31 \%$, si aumenta la temperatura máxima en $1 \%$, entonces el rendimiento de la producción de papa incrementara en $31.91 \%$ y al incremento de las precipitaciones de la lluvia en $1 \%$, entonces el rendimiento de la producción de papa disminuirá en $31.92 \%$; demostrándose de esta manera que los factores determinantes del rendimiento de la producción de la papa son los factores ambientales, y siendo una zona agroecológica que se dedica a la producción de este producto organizo en sus diferentes variedades se hace importante las condiciones del ambiente natural (Tabla 4).

Realizando un análisis a los factores determinantes de la producción de papa, se obtuvo una bondad de ajustado de $97.95 \%$, determinando que el modelo es muy consiste, lo que explica que las variables independientes explican a la producción de papa en $97.95 \%$. Realizando la prueba de significancia individual, estas son menores a $5 \%$, determinando que son significativas e influyen en la variable dependiente (Tabla 4).

Por lo cual, se puede concluir que la temperatura máxima, la temperatura mínima y las precipitaciones de las lluvias determinan en la producción de la papa; explicando de esta manera que ante un incremento de la temperatura en $1 \%$, entonces la producción de la papa incrementara en $31.80 \%$; en el caso de la temperatura máxima, ante un incremento de esta en $1 \%$, entonces la producción de papa aumentara en $30.56 \%$ y si aumenta las precipitaciones de las lluvias en $1 \%$, entonces afectaría de manera negativa en la producción de la papa en $39.95 \%$; lo que es concordante con el rendimiento correspondiente (Tabla 4).

\section{Discusión}

En este sentido, al realizar la discusión, de acuerdo a los resultados obtenidos para el periodo 2000-2018, la producción y rendimiento de la papa tuvo sus altibajos, además, se muestra que las variables climáticas de la temperatura mínima y máxima tienen un efecto positivo sobre el rendimiento y producción de la papa, mientras que las precipitaciones tuvieron un efecto negativo.

Por otro lado, el estudio realizado (32) en el altiplano peruano, indica que los eventos climáticos adversos son muy frecuentes como las heladas y sequias, los cuales son quienes afectan a las actividades productivas como la agricultura y ganadería de manera negativa, presentando un peligro ya que estas son las que velan las principales fuentes de ingreso a muchas familia, cuestión que es coherente con la presente investigación; lo que es concordante con lo que se obtuvo en nuestra investigación, en vista que las variables ambientales determinan en el desarrollo de la actividad agrícola y pecuaria en los andes del Perú. 
Además, los resultados obtenido en este estudio coinciden con los resultados (37), en vista que estas sostienen que en el corto plazo en la producción de papa, el cambio climático llega a tener cierta incidencia sobre la producción de papa. Además, ante aumentos en la temperatura hasta en un $4^{\circ} \mathrm{C}$ los efectos pueden ser positivos en el valor del suelo, lo que implica incrementos en la producción y que hace verificable que el sector agropecuario sea sensible a cambios en el clima y presente mayor sensibilidad cuando se presente la situación de no acceso a servicios básicos y cascos urbanos. En cuanto al enfoque Ricardiano, éste muestra que, en los periodos secos, la temperatura es la variable más afectada el valor del suelo, como consecuencia la variable precipitación no guarda relación con el valor del suelo. Es importante señalar que en el municipio de Villapinzón a nivel rural no hay déficit de agua, debido a que los agricultores planean su producción de acuerdo con las épocas de lluvia, por lo tanto, el riego no es una estrategia determinante para contrarrestar los efectos de cambio climático.

Además, es coherente con lo determinado (38), sobre la producción de cultivos la cual es afectado por el cambio climático, según las observaciones, el efecto de la sequía puede ser mayor o menor según la etapa fenológica; la producción de papa se vio más afectada que la del maíz debido a que las épocas de sequía se encuentran en la etapa de formación de tubérculos, que es la etapa fenológica clave del cultivo. El cultivo de maíz suave disminuye la producción cuando afecte etapas de desarrollo y media, que es cuando se forman los granos.

Los cultivos de papa son extremadamente sensibles a los cambios ambientales (la temperatura tiene un mayor efecto) (39). Por lo tanto, en caso de una temperatura superior a la óptima, el modo de crecimiento se modificará de acuerdo a la duración de las diferentes fases fenológicas, lo que afecta en gran medida el rendimiento logrado, a su vez se ve afectado el número de tubérculos en los calibres mayores y esto se demostró también en la presente investigación.

Complementariamente coincide que, toda vez que en la producción de papa ocurre un efecto producido por la temperatura (30). Es decir, la temperatura promedio más baja para el cultivo de papa es de $8.0^{\circ} \mathrm{C}$ y la más alta es de $11.20^{\circ} \mathrm{C}$. Lo cual indica que el cultivo de papa es uno de los cultivos que tolera esta variación de temperatura y es una buena opción para promover su producción en el centro poblado de Chijichaya del distrito de Ilave-El Collao.

Además, es coherente que la temperatura máxima muestra una tendencia significativa al nivel de un aumento promedio anual de $0.04^{\circ} \mathrm{C} / \mathrm{año}$, hay poca evidencia (0.05), asimismo, la temperatura más baja a nivel de la cuenca mostró un aumento de $0.0004^{\circ} \mathrm{C} /$ año, mientras que Arapa y Progreso mostraron un cambio negativo de 0.01; en la Cuenca de Ramis, la precipitación mostró una tendencia negativa de $0.70 \mathrm{~mm}$ / año, pero no se registró un descenso regional evidente encontrado. Por ello, en los cultivos de papa, el efecto de los días de temperatura y precipitación es significativo, 
un aumento de $83,41 \mathrm{~kg} / \mathrm{ha}$, La precipitación total no tiene un efecto significativo (40).

Finalmente, coincide con los resultados arrojados en este estudio (28), donde pudo demostrar que el efecto del cambio climático a través de mapas temáticos, para las áreas de las cuencas de Huancané y Coata, donde también se esperó que la temperatura promedio aumente en $3{ }^{\circ} \mathrm{C}$. La zona más afectada corresponderá a la localidad de Huancané, debido a las condiciones locales y climáticas, la escasez de precipitaciones, además de futuras reducciones de precipitaciones. Se evidencia que, en los próximos 2071-2100 años, en comparación con el cultivo de quinua, el cultivo de papa en la región de Huancané se verá afectado por una mayor demanda de riego agrícola, que se debe a la reducción de las lluvias y el clima local y regional. En términos de temperatura, la temperatura máxima y mínima prevista en el futuro no superará la temperatura crítica para el cultivo de papa y quinua.

\section{CONCLUSIONES}

Las variables climáticas como la temperatura mínima, la temperatura máxima y las precipitaciones si influyen en el rendimiento y la producción de papa, los cuales explican en $97 \%$. Además, se puede decir que dichos factores climáticos no son los únicos que afectan o influyen en la producción, debido a que existen otras variables tales como; tierra, mano de obra, tecnología, entre otros.

Además, el factor climático que más influye en el rendimiento y la producción de papa es la temperatura mínima, esto teniendo en consideración un nivel de significancia al $5 \%$. Además, se sabe que la papa tiene facultades adaptativas a condiciones adversas del clima, debido a que, cuanto mayor sean las temperaturas máximas, la producción aumentara. Sin embargo, cuando las temperaturas máximas excedan, la producción se verá afectada negativamente. Del mismo modo, a mayores temperaturas mínimas existirá mayor producción, pero, una vez superada la temperatura mínima optima provocará que la producción se vea afectada (disminuya), además, altos niveles de precipitación pluvial disminuirán la producción por excesivas precipitaciones que perjudicando a la producción.

Por todo lo expuesto, en un contexto de cambio climático en la región de Puno, se requiere fortalecer las ventajas comparativas que posee la papa andina que se cultiva desde tiempos atrás y, ser una fuente de ingresos económicos en los agricultores de bajos recursos y a su vez es un el alimento básico en la canasta de consumo de los agricultores del altiplano. La reducción del rendimiento de la producción de papa puede tener un efecto negativo en el bienestar de la población rural.

\section{REFERENCIAS BIBLIOGRÁFICAS}

1. Caldeira CF, Giannini TC, Ramos SJ, Vasconcelos S, Mitre SK, Pires JP de A, et al. Sustainability of Jaborandi in the eastern Brazilian Amazon. Vol. 15, Perspectives in Ecology and Conservation. Associacao Brasileira de Ciencia Ecologica e Conservacao; 2017:161-71 
2. Daccache A, Weatherhead EK, Stalham MA, Knox JW. Impacts of climate change on irrigated potato production in a humid climate. Agric For Meteorol. 2011 Dec 15;151(12):164153

3. Eamus D. The interaction of rising $\mathrm{CO} 2$ and temperatures with water use efficiency. Plant Cell Env. 1991;14(8):843-52.

4. Engelbrecht FA, Landman WA, Engelbrecht CJ, Landman S, Bopape MM, Roux B, et al. Multiscale climate modelling over southern Africa using a variable-resolution global model. Water SA. 2011;37(5):647-58

5. Rosenzweig C, Phillips J, Goldberg R, Carroll J, Hodges T. Potential impacts of climate change on citrus and potato production in the US. Agric Syst. 1996 Dec 1;52(4):455-79

6. Van Der Waals JE, Krüger y K, Franke AC, Haverkort AJ, Steyn JM, Krüger K. Climate Change and Potato Production in Contrasting South African Agro-Ecosystems 3. Effects on Relative Development Rates of Selected Pathogens and Pests. Potato Res. 2013;56:6784

7. Balboa Navarro I. Tierras y azúcar. Las transformaciones agrarias y el ascenso de la plantación en Cuba. Investig Hist Econ. 2015 Feb 1;11(1):43-51

8. Marmolejo D, Ruiz J. Tolerancia de papas nativas (Solanum spp.) a heladas en el contexto de cambio climático. Sci Agropecu [Internet]. 2018 Oct 2 [cited 2021 Sep 10];9(3):393-400. Available from: https://revistas.unitru.edu.pe/ index.php/scientiaagrop/article/view/2085

9. Navas. El cambio climático y la agricultura: un desafío para Colombia y el mundo. instnameUniversidad los Andes. 2011

10. Pinzón Colmenares IE, Ramírez Cando LJ. Ecoeficiencia de los modelos de producción agrícola de maíz duro y su influencia al cambio climático en Shushufindi Ecuador. LA GRANJA Rev Ciencias la Vida [Internet]. 2021 Feb 1 [cited 2021 Sep 10];33(1):76-91. Available from: http:// scielo.senescyt.gob.ec/scielo.php?script=sci_ arttext\&pid=S1390-85962021000100076\&lng= es\&nrm=iso\&tlng=es
11. Reyes Anistro Gl. Evaluación de vulnerabilidad ante variabilidad climática del maíz (Zea Mays) en el Distrito de Desarrollo Rural - 073, Toluca [Internet]. 2017 [cited 2021 Sep 10]. Available from: https://core.ac.uk/ reader/154798100

12. Hijmans RJ. Global distribution of the potato crop. Am J Potato Res. 2001;78(6):403-12

13. Sanabria J, Lhomme JP. Climate change and potato cropping in the Peruvian Altiplano. Theor Appl Climatol 20121123 [Internet]. 2012 Sep 18 [cited 2021 Sep 10];112(3):68395. Available from: https://link.springer.com/ article/10.1007/s00704-012-0764-1

14. Sánchez Castañeda J. Mercado de productos agrícolas ecológicos en Colombia. Suma Negocios. 2017 Jul 1;8(18):156-63

15. Urbina DA, Quispe MR. La pobreza monetaria desde la perspectiva de la pobreza multidimensional: el caso peruano [Internet]. Vol. 2, Enfoque n. os. 2016 [cited 2020 Dec 14]. Available from: http://repositorio-anterior. ulima.edu.pe/handle/ulima/5815

16. Ocampo O. El cambio climático y su impacto en el agro Climate Change and its Impact on the Agriculture. Rev Ing. 2011 Jul;0(33):115-23.

17. Ramírez L, Mostacero J, López E, Cruz AD La, Gil A. Aspectos etnobotánicos de Cuspón, Perú: Una comunidad campesina que utiliza 57 especies de plantas en sus diversas necesidades. Sci Agropecu [Internet]. 2020 Apr 1 [cited 2021 Sep 10];11(1):7-14. Available from: https://revistas.unitru.edu.pe/index.php/ scientiaagrop/article/view/2799

18. Benoit GR, Stanley CD, Grant WJ, Torrey DB. Potato top growth as influenced by temperatures. Am Potato J. 1983 Jul;60(7):489501

19. Burt RL. Influence of short periods of low temperature on tuber initiation in the potato. Eur Potato J. 1964 Dec;7(4):197-208

20. Bindi $M$, Hacour $A$, Vandermeiren K, Ojanperä K, Selldén G, Högy $P$, et al. Chlorophyll concentration of potatoes grown under elevated carbon dioxide and/ 
or ozone concentrations. Eur J Agron. 2002 Nov;17(4):319-35

21. Pari JGZ, Mamani FE, Rojas P V., Landaeta JCZ. Evaluación de substratos y producción de Beauveria Brongniartii (sacc.) petch para control de gorgojo de los andes (premnotrypes spp) en cultivo de papa. Rev Investig Altoandinas [Internet]. 2015 [cited 2021 Sep 10];17(3):15. Available from: https://dialnet. unirioja.es/servlet/articulo?codigo $=5294161$

22. Bezemer TM, Jones TH, Knight KJ. Longterm effects of elevated $\mathrm{CO} 2$ and temperature on populations of the peach potato aphid Myzus persicae and its parasitoid aphidius matricariae. Oecologia. 1998 Aug;116(12):128-35

23. Cao W, Tibbitts TW. Starch concentration and impact on specific leaf weight and element concentrations in potato leaves under varied carbon dioxide and temperature. J Plant Nutr. 1997;20(7-8):871-81

24. Awmack CS, Woodcock CM, Harrington R. Climate change may increase vulnerability of aphids to natural enemies. Ecol Entomol. 1997;22(3):366-8

25. Haverkort AJ, Franke AC, Engelbrecht FA, Steyn JM. Climate Change and Potato Production in Contrasting South African Agroecosystems 1. Effects on Land and Water Use Efficiencies. Potato Res 2013561 [Internet]. 2013 Mar 7 [cited 2021 Sep 10];56(1):31-50. Available from: https://link.springer.com/ article/10.1007/s11540-013-9230-4

26. Elsgaard L, Børgesen CD, Olesen JE, Siebert $S$, Ewert $F$, Peltonen-Sainio $P$, et al. Shifts in comparative advantages for maize, oat, and wheat cropping under climate change in Europe. Food Addit Contam Part A. 2012 Oct;29(10):1514-26

27. Temmerman L, Hacour A, Guns M. Changing climate and potential impacts on potato yield and quality 'CHIP': introduction, aims and methodology. Eur J Agron. 2002 Nov;17(4):23342
28. Torres López SKE. Impacto de cambio climático en el cultivo de papa (Solanum tuberosum) y quinua (Chenopodium quenoa Will.) en el departamento de Puno [Internet]. Universidad Nacional Agraria La Molina. Universidad Nacional Agraria La Molina; 2016 [cited 2021 Sep 10]. Available from: http://repositorio.lamolina.edu.pe/handle/ UNALM/2581

29. MINAGRI. Plan de Gestión de Riegos y Adaptación al Cambio Climático demandara S/. 1,950 millones hasta el 2021. 2012

30. Lima Atencio YW. Efecto de cambios climatológicos en la producción de papa en centro poblado Chijichaya - Ilave [Internet]. Universidad Nacional del Altiplano. Universidad Nacional del Altiplano; 2015 Dec [cited 2021 Sep 10]. Available from: http:// repositorio.unap.edu.pe/handle/UNAP/4609

31. Tonconi J. Producción agrícola alimentaria y cambio climático: un análisis económico en el departamento de Puno, Perú Food production agriculture and climate change: An economic analysis in the Department of Puno, Peru. Idesia . 2015;33(2):119-36

32. Aliaga S, Terrazas F, Ortuño N. Estrategias ecológicas para el manejo del tizón tardío de la papa [Phytophthora infestans (Mont.) De Bary]. Rev Latinoam la Papa [Internet]. 2017 Jun 9 [cited 2021 Sep 10];21(1):1-14. Available from: http://ojs.papaslatinas.org/index.php/ rev-alap/article/view/261

33. Cabrera Hoyos HA, Gastelo Benavides M, Otiniano Villanueva $R$, Pacheco del Castillo MÁ, Janampa A. Variedades de Papa Resiliente al Cambio Climático, para la Seguridad Alimentaria del Perú [Internet]. Instituto Nacional de Innovación Agraria. Instituto Nacional de Innovación Agraria; 2020 [cited 2021 Sep 10]. Available from: http://repositorio. inia.gob.pe/handle/20.500.12955/1250

34. Haan $S$ de, Burgos G, Ccanto R, Arcos J, Scurrah M, Salas E, et al. Effect of production environment, genotype and process on 
the mineral content of native bitter potato cultivars converted into white chuño. J Sci Food Agric [Internet]. 2012 Aug 15 [cited 2021 Sep 10];92(10):2098-105. Available from: https:// onlinelibrary.wiley.com/doi/full/10.1002/ jsfa.5589

35. Lavado Casimiro WS, Ronchail J, Labat D, Espinoza JC, Guyot JL. Basin-scale analysis of rainfall and runoff in Peru (1969-2004): Pacific, Titicaca and Amazonas drainages. Hydrol Sci J [Internet]. 2012 May 3 [cited 2021 Feb 25];57(4):625-42. Available from: https://www. tandfonline.com/doi/full/10.1080/02626667.2 012.672985

36. Hernández, Fernández, Baptista. Metodología de la investigación [Internet]. McGraw Hill., editor. Mexico D.F; 2014. Available from: https://dspace.scz.ucb.edu.bo/ dspace/bitstream/123456789/166/1/1646.pdf

37. González, K. y Galera K. Efectos del cambio climático sobre la producción de papa en el municipio de Villapinzón (CundinamarcaColombia) a partir del Enfoque Ricardiano. Rev Investig Agrar y Ambient. 2014;5(1):231
38. Pilco P. Impacto del cambio climático en la producción de papa y maíz en la comunidad de Pululahua. 2017;88

39. Martín Martín R, Jerez Mompie E. Efecto de las temperaturas en el rendimiento de la papa (Solanum tuberosum L.) variedad romano. Cultiv Trop. 2017;38(1):75-80

40. Tonconi Quispe J. Producción agrícola alimentaria y cambio climático: un análisis económico en el departamento de Puno, Perú. Idesia (Arica) [Internet]. 2015 [cited 2021 Sep 10];33(2):119-36. Available from: http://www.scielo.cl/scielo.php?script=sci_ arttext\&pid=S0718-34292015000200014\&lng= es\&nrm=iso\&tlng=n 